\title{
Coulomb effect on the dynamics of atoms in a strong elliptical laser field: Unification of the excitation and ionization
}

\author{
Xiang $\mathrm{Gao}^{1, \text { * }}$ and Xiao-Min Tong ${ }^{1,}$ † \\ ${ }^{1}$ Center for Computational Sciences, University of Tsukuba, \\ 1-1-1 Tennodai, Tsukuba, Ibaraki 305-8573, Japan
}

\begin{abstract}
We investigate the Coulomb effect on the dynamics of atoms in a strong elliptical laser field by solving the three-dimensional time-dependent Schrödinger equation with and without the photoelectron core Coulomb interactions and find that the Coulomb effect plays an important role for the excitation and ionization processes while it is less important for the high-order harmonic generation. Our calculation results can serve as a benchmark for other approximative methods. Looking at the photoelectron momentum distribution, we find that the Coulomb effect is important for the momentum distribution in the polarization plane and less important for the momentum distributions along the normal direction perpendicular to the polarization plane. Similar to the photoabsorption in a weak field, we demonstrate that the excitation and ionization can be treated in a unified way, which implies the low energy photoelectron distributions can be connected with the populations of highly excited states.
\end{abstract}

\section{INTRODUCTION}

The interaction of light with atoms is of a great scientific interest in understanding the response of matter to an external electromagnetic field. Ionization and excitation are essential parts of photoabsorption. For a weak field, the electron external interaction can be treated perturbatively and atomic structures play an important role. It is well-known that the discrete absorption spectrum of an atom has intimate relations with the photo-ionization spectrum in the continuum energy region, which is determined in essence by the long-range Coulomb interaction [1. Such understanding greatly simplified the theoretical description of the weak electromagnetic interaction with atoms and is the foundation of the multi-channel quantum defect theory [2], in which the excitation and ionization can be treated in a unified way.

In contrast, when the external electromagnetic field becomes strong, perturbative theory fails. Many unique aspects of intense light-matter interaction at atomic and molecular levels have been discovered over the last few decades, including the above-threshold ionization (ATI) [5, 6], high-order harmonic generation (HHG) [7-9, and so on. These phenomena are later found to be very important to many technological breakthroughs and attract many research interests. For example, ATI process can be used to image the atomic or molecular structures [10 12, and the HHG process is crucial for converting an infrared (IR) laser into a soft x-ray laser [13] or attosecond pulse [14, 15].

In view of these activities, the development and application of different complementary theoretical methods for the analysis of laser-driven nonlinear processes

\footnotetext{
* xgao@csrc.ac.cn

$\dagger$ tong.xiaomin.ga@u.tsukuba.ac.jp
}

in atoms and molecules is of a great interest. It has been generally accepted that most of the physical processes for atoms in a strong field can be understood by the three-step model [16] or explained by the strong field approximation (SFA) [17 20, in which the photoelectron parent core Coulomb interaction is ignored. Some more quantitative approaches include, among others, approximative but powerful multi-photon and tunneling ionization formulas (ADK theory with its variants) [21 26], non-perturbative Floquet methods [27, 28], semiclassical approximation methods 29, classical-trajectory Monte Carlo simulations 30, 31, as well as ab initio numerical methods to solve the time-dependent Schrödinger equation (TDSE) 32 39. The TDSE method should be the most accurate one for the description of strong field interaction with atoms. However, even for the simplest one-electron hydrogen atom which can be studied analytically for the ground and excited states without an external field, its interaction with a strong laser field, especially with an elliptical laser field, cannot be treated easily even with a modern computer. Because the electron moving over a large distance can still be driven back to the parent core by the laser field, we have to describe the electron motion in a large space numerically. For an atom in a linearly polarized laser field, due to the cylindrical symmetry, it is a two-dimensional (2D) time-dependent problem while for atoms in an elliptical field, it is a three dimensional (3D) time-dependent problem. This makes the numerical simulation more difficult. Thus, many previous TDSE studies were mainly focused on solving $2 \mathrm{D}$ TDSE 32 37, which limits our knowledge of the strong field atom interactions. Recently, combined with a second order-split-operator method [40, we extended our 2D generalized pseudo-spectral time-dependent method 37. to a 3D time-propagator 39. With this generalized 3D time-propagator, in principle, we can study most dynamical processes of atoms in any kind of time-dependent 41] or time-independent external fields [42, 43]. 
We note that in most of the above mentioned qualitative and approximated quantitative theories, the photoelectron parent core Coulomb interaction is completely ignored. Recently, more detailed measurements [4446] showed that the ignored Coulomb interaction plays an important role for the low energy ATI spectra 47 [50]. Then it is natural to raise the question on whether the long-range Coulomb interaction in the strong field physics would play a similar role as that in the weak field case. As the 3D-TDSE calculations can serve as a benchmark for other approximative methods, it is interesting to make a comparative study on the role played by the longrange Coulomb potential in the non-perturbative lightatom interactions. In this work, we will make a comprehensive study on the interaction between a hydrogen atom and a strong elliptical laser field. We will mainly focus on the Coulomb effect and the relations between the photoexcitation and ionization of atoms in a strong field.

\section{THEORETICAL METHOD}

The dynamic properties of hydrogen atoms in an external field can be obtained by solving the time-dependent Schrödinger equation in three dimensions (TDSE3D). Based on the second order-split-operator method [40] and the generalized pseudo-spectral (GPS) method [37, we have developed a three-dimensional time-propagator for solving the TDSE3D [39]. This method takes the advantage of the split-operator method and GPS method to greatly reduce the mesh points in $3 \mathrm{D}$ while capturing all the physical details. The theoretical approach and computational procedure have been published elsewhere [39], only the essentials are presented here to facilitate later discussions (atomic units $\hbar=m_{e}=e=1$ are used unless hereafter specified otherwise).

The TDSE3D can be expressed as,

$$
i \frac{\partial}{\partial t} \Psi(\mathbf{r}, t)=\left[H_{0}+V_{\text {ext }}(\mathbf{r}, t)\right] \Psi(\mathbf{r}, t)
$$

with $H_{0}=-\nabla^{2} / 2+V_{0}(r)$, the external field-free Hamiltonian of an atom, $V_{\text {ext }}(\mathbf{r}, t)=-\mathbf{r} \cdot \mathbf{E}(t)$ the electron external field interaction in length gauge under the dipole approximation and $\mathbf{r}$ the electron coordinate. $V_{0}(r)$ is the atomic potential with the single active electron approximation and it can be calculated with the density functional theory using a local density approximation with a self-interaction correction 51. For hydrogen atoms, $V_{0}(r)=-1 / r . \mathbf{E}(t)$ is the electric field of an elliptical laser, which is generally expressed as,

$$
\mathbf{E}(t)=\frac{f(t) E_{0}}{\sqrt{1+\epsilon^{2}}}\left(\hat{x} \cos \omega_{0} t+\hat{y} \epsilon \sin \omega_{0} t\right)
$$

with $\hat{x}$ the unit vector along the $x$-direction (major axis), $\hat{y}$ the unit vector along the $y$-direction (minor axis), $f(t)$ the pulse envelop function, $E_{0}$ the laser peak field strength, $\omega_{0}$ the laser center frequency and $\epsilon$ the ellipticity. We chose the $x y$-plane as the polarization plane. The time-dependent wavefunction $\Psi(\mathbf{r}, t)$ can be expanded in the partial wave basis as,

$$
\Psi(\mathbf{r}, t)=\sum_{i, l, m} C_{i, l, m}(t) R_{i l}(r) Y_{l m}(\theta, \varphi)
$$

where $R_{i l}(r) Y_{l m}(\theta, \varphi)$ is the eigen state of the field free atomic Hamiltonian $H_{0}$ in a finite box with $R_{i l}(r)$ the radial eigen wavefunction obtained with the GPS grids [37] and $Y_{l m}(\theta, \varphi)$ the spherical harmonic function. Since the numerical simulations are carried out in a finite box, both the physical bound and continuum states can be represented by $R_{i l}(r) Y_{l m}(\theta, \varphi)$ basis.

For the ATI calculations, we separate the finite box into an inner and outer region to avoid the unphysical reflection from the boundary. When the time-dependent wave-function moves into the outer region, we project the wave-function onto momentum space (atomic Coulomb Volkov state) to extract the ionization information and then remove it from the wave-function in real space as discussed in [52]. The numerical convergence was checked by changing the box size, time steps, the number of partial waves and so on. For the highest laser intensities $2 I_{0}$ with $I_{0}=10^{14} \mathrm{~W} / \mathrm{cm}^{2}$ of $800 \mathrm{~nm}$ wave length and $20 \mathrm{fs}$ full pulse duration used in the present study, we chose the maximum radial size of the box 600 a.u., a timestep of 0.1 a.u. and 256 partial waves. With $\Psi(\mathbf{r}, t)$, all the physical observables, such as the photoelectron momentum spectra, photoexcitation distribution, and HHG spectra, can be calculated. Note that although the box size is comparable with the laser wavelength, since the photoelectron moves mainly the plane perpendicular to the laser propagation direction, the dipole approximation is still valid here.

For photoionization process, we obtain the momentum distribution

$$
\frac{d^{3} P(\mathbf{p})}{d p_{x} d p_{y} d p_{z}}
$$

as discussed in [52]. The ATI spectra can then be calculated as,

$$
\frac{d P(E)}{d E}=\int \frac{d^{3} P(\mathbf{p})}{d p_{x} d p_{y} d p_{z}} \delta\left(\frac{p^{2}}{2}-E\right) d^{3} \mathbf{p},
$$

with $E$ the photoelectron energy. The transition probability to a given bound excited state $(n, l)$ can be directly obtained as

$$
P_{n, l}=\sum_{m=-l}^{l}\left|C_{n, l, m}(t \rightarrow \infty)\right|^{2}
$$

at the end of the laser pulse. Note that we define the bound states $(n, l)$ here for their field free eigen energy in the finite box $E_{n, l}<0$, as conventions, eg [38]. The total excitation probability for bound states can be obtained as

$$
P_{e}=\sum_{n, l} P_{n, l},
$$


and the total ionization probability is

$$
P_{i}=\int \frac{d P}{d E} d E
$$

The HHG power spectra can be calculated from the Fourier transform of the time-dependent induced dipole moment as discussed in Ref. 37.

In the SFA [17 20], the interaction between the photoelectron and the laser field is fully taken into account, but the Coulomb interaction between the photoelectron and ion core is ignored. This approximation can be represented by a TDSE3D calculation with a model potential without the long-range Coulomb intertaction. Therefore, to mimic the SFA numerically and identify the role of the long-range Coulomb potential, we also investigate the dynamics with a model potential as

$$
V_{0}(t)= \begin{cases}-\frac{1}{r} & \text { for } r \leq R_{c} \\ -\frac{1}{r} e^{-\alpha\left(r-R_{c}\right)^{2}} & \text { for } r>R_{c} .\end{cases}
$$

The criteria to choose the model potential or the parameters is to remove the long-range Coulomb potential without affecting the ground state wavefunction. We call this set of simulation results "without Coulomb interaction", which can represent the SFA results under the same condition. In the present simulation, we used $\alpha=1, R_{c}=10$ a.u., where only the bound states up to $n=3$ can be described reliably.

\section{RESULTS AND DISCUSSION}

Based on the above method, we investigated the dynamical processes of hydrogen atoms in an elliptical strong laser field. We chose laser parameters with the center wavelength $800 \mathrm{~nm}$, full pulse duration of $20 \mathrm{fs}$.

Figure 1 shows the total excitation and ionization probabilities. In Fig. 1 (a), the excitation probabilities increase with the increasing laser intensities at a given ellipticity. For a given laser intensity, the probabilities decrease dramatically by at least four orders of magnitude from linearly $(\epsilon=0)$ to circularly $(\epsilon=1)$ polarized cases. This can be understood from the differences in the selection rule of optical transitions for different ellipticities. For a linearly polarized light, the electron can be excited to Rydberg states $(n, l)$ with various angular momentum (in favor of relatively low angular momentum states), whereas for a circularly polarized light, the electron can only go to a circular state $|m|=l$ with much higher angular momentum $l$. As is well known that there are only very limited number of bound states for a short-range interaction potential, the results without the Coulomb interaction are orders of magnitude lower [the green curve vs the red one in Fig. [1(a)].

Figure 1(b) shows the results of total ionization probability. Compared with the excitation probability, the total ionization probability decreases much slower with the increasing ellipticities, and the influence of long-range
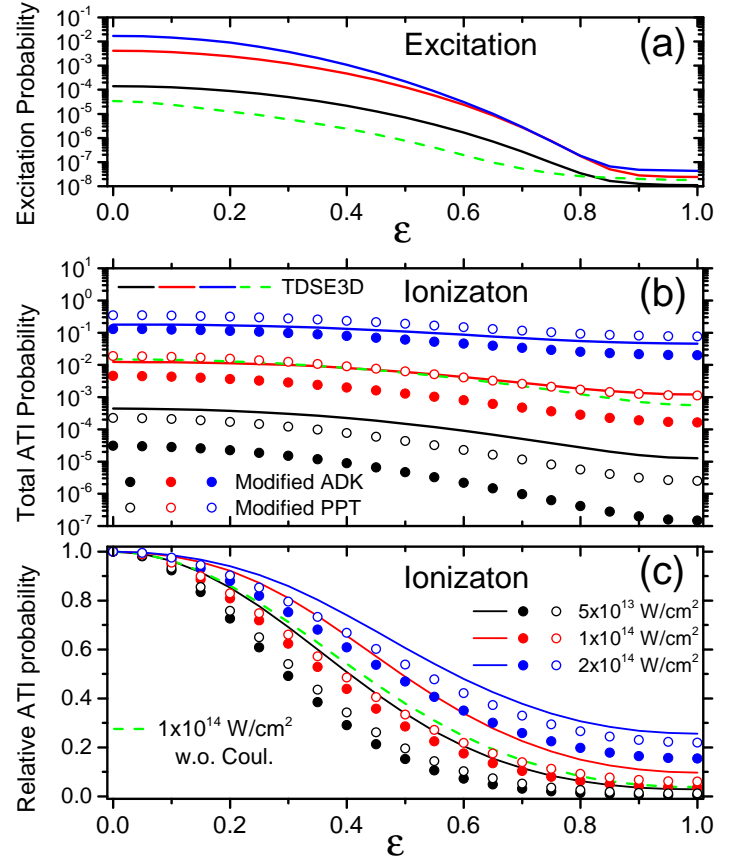

FIG. 1. (Color online) Total excitation (a) and ionization (b) probabilities of hydrogen atoms in strong elliptical laser fields as a function of the ellipticity for three laser intensities $0.5 I_{0}$ (black, upper), $I_{0}$, (red, middle) and $2 I_{0}$ (blue, lower), respectively. The normalized ionization probabilities to $\epsilon=0$ is replotted in (c). The dashed (green) ones stand for the results without Coulomb interaction for $I_{0}$. Other laser parameters are $800 \mathrm{~nm}$ of center wavelength, $20 \mathrm{fs}$ of the full laser pulse duration.

Coulomb interaction is also smaller. The decrease of the ionization probabilities with the increasing ellipticity can be attributed to the decrease of the peak field strength as shown in Eq. (2), since the tunneling ionization is sensitive to the peak field strength.

Our full TDSE3D calculation results can also serve as a benchmark for other approximated theories, such as the widely used ADK 23] and PPT 22] models. It's worth comparing our results with these models. For strong field ionization processes, one usually uses the Keldysh parameter 21] to characterize different ionization mechanisms. Here the Keldysh parameter is defined as $\gamma=\omega_{0} \sqrt{2 I_{p}} / E_{0}$, where $\omega_{0}$ is the angular frequency of the laser field, $I_{p}$ is the ionization potential of the atom and $E_{0}$ is the laser peak field strength. When $\gamma<1$ the static tunneling process is dominant, where the adiabatic approximation in the ADK theory [23] is valid. In this tunneling ionization region, we can further define a critical field $E_{b}=\left(2 I_{p}\right)^{2} / 16 Z_{c}$ with $Z_{c}$ the charge of the parent core. Above this critical field, we enter the barrier-suppression region and the tunneling ionization rate based on the perturbative ADK theory would overestimate the exact ionization rate 26 . When $\gamma>1$ the multiphoton process is dominant, where the non-adiabatic effect [53] is important. The PPT theory 


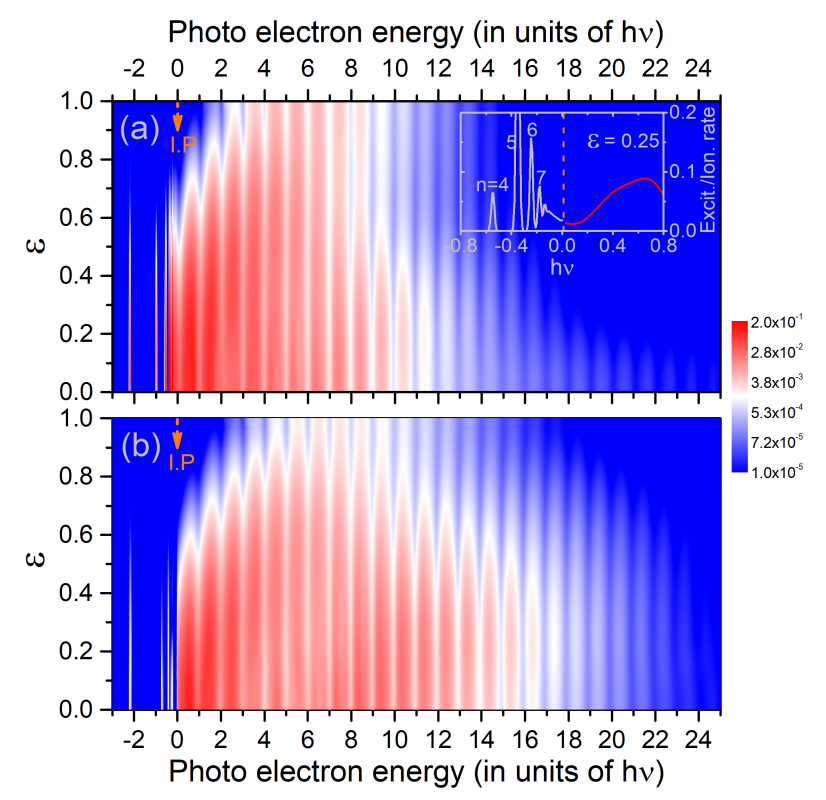

FIG. 2. (Color online) ATI and excitation spectra of $\mathrm{H}$ atoms in elliptical laser fields as a function of the ellipticity with (a) and without (b) the Coulomb interaction for laser intensity of $I_{0}$. The insertion exhibits the smooth connection of the excitation and ionization spectra (i.e., the oscillator strength density) near the ionization threshold for $\epsilon=0.25$. Other laser parameters are the same as the ones in Fig. 1 .

22] can be viewed as an extension to the ADK theory to take into account the non-adiabatic effect, which can also maintain the form of the ADK theory in the tunneling region. For the present laser intensities of $0.5 I_{0}, I_{0}$ and $2 I_{0}$, the corresponding Keldysh parameters are 1.509, 1.067, 0.754 , with the $E_{0} / E_{b}$ about $0.6,0.85$ and 1.2 , respectively, which in fact covers all typical regions of strong field ionization processes. The modified ADK theory [26] which remedies the error in the barrier-suppression region, and the modified PPT theory with an updated Coulomb correction [54] are chosen for the comparisons. As can be seen in Fig. 1(b), the TDSE3D results are all larger than those of the modified ADK theory [26]. With a higher laser intensity, the differences between the two results become smaller, which agree with the increasing validity of the adiabatic approximation. On the other hand, there is a great improvement for the $0.5 I_{0}$ in the result of the modified PPT theory [54, which demonstrates the importance of the non-adiabatic effects in this multi photon region. For the $I_{0}$ case, it is interesting to note that the result of the modified PPT theory is in better agreement with our TDSE3D result including the longrange Coulomb interactions, which demonstrates the correctness of the new Coulomb correction used in the modified PPT theory [54. However, for the $2 I_{0}$ case, the result from the modified PPT theory is overestimated compared to our results and the modified ADK theory [26, which indicates the barrier suppression effect is important for this case. Therefore, a further improvement of the modified PPT theory can be made by incorporating the barrier-suppression correction used in the modified ADK theory [26]. To see the details, we also plot the relative ionization probability with respect to that of the linear polarization in Fig. 1(c). We found that although the modified PPT results 54 are in closer agreement with the TDSE3D results, both the modified ADK results 26 and the modified PPT results [54, as well as the TDSE3D results without the Coulomb interaction are smaller than the corresponding TDSE3D results for large ellipticities.

Let's return to discuss the spectra of photon absorption processes in the strong field. In a weak field limit, the photon absorption is dominated by a single photon process, where the optical selection rule applies. The final excited or continuum states with the same angular momentum $l$ form a channel. The photoionization probability is proportional to the oscillator strength density $d f^{l} / d E$. Below the ionization threshold, the photoexcitation probability is proportional to the oscillator strength $f^{l}$. The relation between the two physical quantities is [1],

$$
\frac{d f^{l}}{d E}=f^{l} \frac{d n^{l}}{d E}
$$

where $d n^{l} / d E$ is the density of state for a given channel $l$ and a given state. This relation is a natural consequence of the properties of the wavefunctions in a long-range Coulomb potential [2]. Therefore, the photo-excitation and photoionization can be treated in a unified way. In experiments, the energy resolution is finite, so the measured oscillator strength is actually the oscillator strength density near the threshold, which is smoothly connected to the photoionization spectra [1, 55]. In the numerical calculation of the oscillator strength density $d f^{l} / d E$ using a basis set, we have shown that it can be calculated by two equivalent methods [55]. One is to calculate the density of state directly by finite difference (including the pseudo states) and using Eq. (10 to obtain $d f^{l} / d E$. The other is to convolute the calculated $f^{l}$ with a finite line width. Since the second one is more straightforward for calculations, especially in complex cases, we use it in the present study.

For strong laser field cases, the photoabsorption processes are highly nonlinear, in which many photons can be absorbed and the optical selection rule does not exist. The final state contains many angular momentums. However, we still can extend Eq. 10 to relate the excitation probability and ATI probability as,

$$
\frac{d P}{d E}=\sum_{n, l} P_{n, l} \frac{d n^{l}}{d E},
$$

where $d P / d E, P_{n, l}$ are the ionization and excitation probabilities, respectively.

Figure 2(a) displays our calculated $d P / d E$ both below and above the ionization threshold. We see clearly that $d P / d E$ in the discrete energy region is smoothly connected with the one in the continuum energy region for 
The nomalized yield distribution

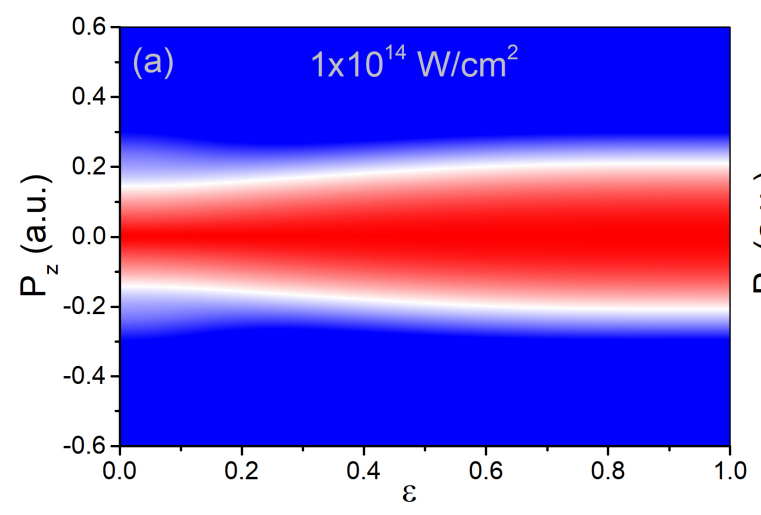

The nomalized yield distribution

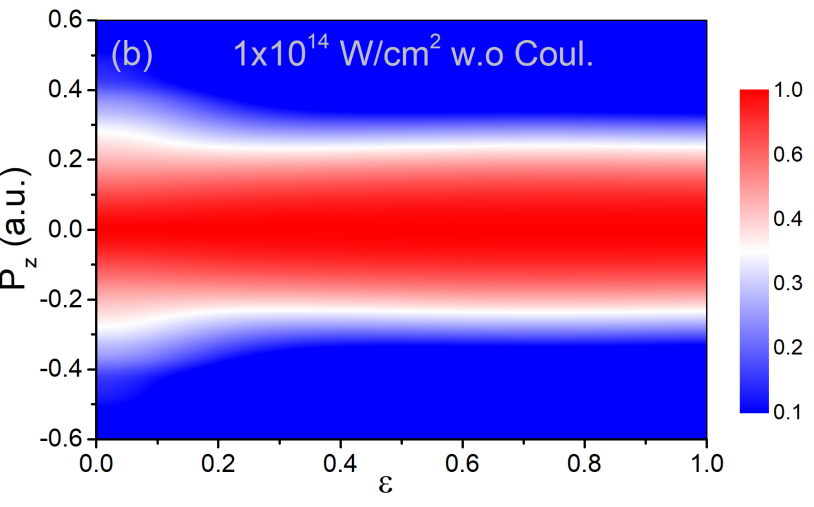

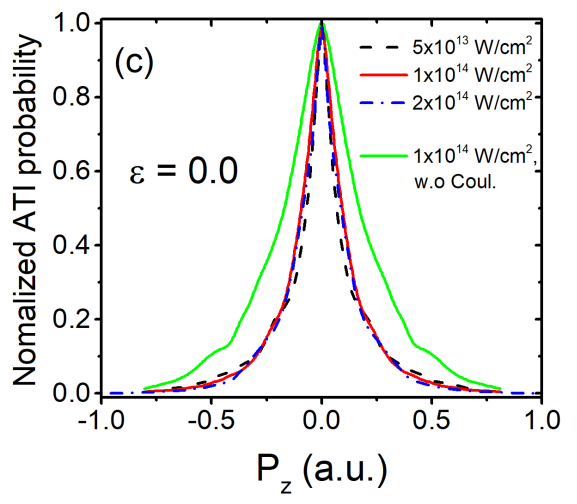
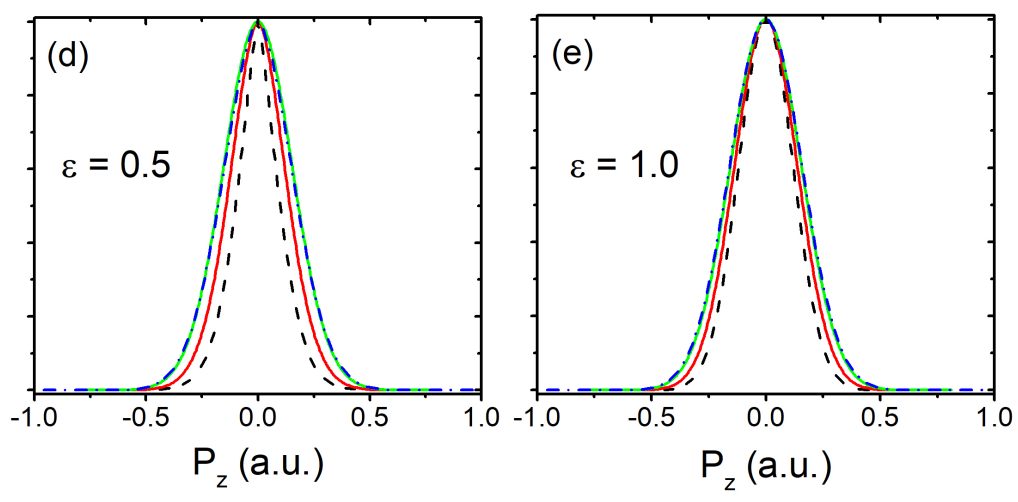

FIG. 3. (Color online) Normalized electron momentum distribution in the direction perpendicular to the polarization plane for $\mathrm{H}$ atoms in elliptical laser fields as a function of the ellipticity with (a) and without (b) Coulomb interaction for laser intensity of $I_{0}$. (c)-(e) are the comparisons of three intensities, $0.5 I_{0}$ (dashed black), $I_{0}$ (solid red), and $2 I_{0}$ (dash dotted blue) for a given ellipticity, respectively. The spectra without including Coulomb interaction at $I_{0}$ is also plotted (solid green). Other laser parameters are the same as the ones in Fig. 1

all ellipticities, which can be viewed as a continuation of the ATI spectra. As indicated in Fig. 1(a), the excitation probabilities decrease by serval orders of magnitude from the linear to circular polarizations. But for each ellipticity, $d P / d E$ is found to connect with the ATI spectra smoothly as shown in Fig. 2(a). We show a zoom-in spectra near the ionization threshold in the insertion of Fig. 2(a) for a typical case with $\epsilon=0.25$. As a comparison, we also show the results without the Coulomb interaction in Fig. 2(b). In this case, the $d P / d E$ is not a continuum function at the ionization threshold, which demonstrates that the long-range Coulomb interaction is the cause of the continuation property shown in Fig.2(a). Considering the ATI process, the electron moving over a large distance can still be driven back to the parent core by the laser field, these differences indicate the importance of the long-range atomic Coulomb interaction during electron motions. The present study shows that there is still an intimate relation between the strong field excitation and ionization in the presence of the atomic long-range Coulomb interaction, which allows us to treat the low energy ATI process and the excitation process in a unified way.
There are some recent interests in the vertical photoelectron momentum distribution of atoms in a strong laser field [53, 56, 61. According to the tunneling ionization ADK theory 62, the vertical photoelectron momentum distribution is substantially influenced by the intensity of the laser. More specifically, the drifted electron momentum in the polarization plane would increase with the field amplitude, and the widths of the vertical momentum distributions both in and perpendicular to the polarization plane would increase with the square root of the field amplitude. It was reported [56 58] that one can calibrate the peak intensity of the circularly polarized laser by comparing the ADK predicted momentum distribution with the measured one in the polarization plane, where the statistical error bar can be a few percents [58. Please note such small statistical error bar indicates the method is very precise, but may not be "accurate" due to the possible systematic errors caused by the non-adiabatic effects [63. Arissian et al [57] also demonstrated experimentally the possibility to use the widths of the vertical momentum distributions in the direction perpendicular to the polarization plane for calibrating the laser peak intensity. However, there is a sys- 
tematic difference therein between the measurement and the ADK theory. Hofmann et al 53 carried out further numerical TDSE studies and demonstrated that while the width of the vertical momentum distribution on the polarization plane from the ADK theory was in agreement with the TDSE calculation, the drifted momentum used in 57] for intensity calibration was not. Although they showed that by considering the non-adiabatic effect the difference will be substantially reduced, there remains a quantitative difference. We note that the studies of the transverse momentum distribution in the direction perpendicular to the polarization plane are still limited. Ivanov et al 59 61] have studied the evolution of such distribution with varying ellipticity under a single laser pulse intensity. Thus, we will carry out a more systematical investigation on the $1 \mathrm{D}$ momentum distribution in the direction perpendicular to the polarization plane defined as

$$
\frac{d P\left(p_{z}\right)}{d p_{z}}=\int \frac{d^{3} P(\mathbf{p})}{d p_{x} d p_{y} d p_{z}} d p_{x} d p_{y} .
$$

The normalized (to $p_{z}=0$ ) 1D momentum distributions are shown in Fig. 3. The $1 \mathrm{D}$ momentum distribution shows an interesting variation with the increasing ellipticity 39, which is opposite with the expectation of the tunneling ionization model 62. As can be seen, the long-range Coulomb interaction has a significant influence on the 1D distribution, especially for the linear polarization. This should originate from the Coulomb focusing effect, which makes the final lateral distribution at the asymptotic region narrower than the one at the tunneling exit point $39,60,64$. We see that this distribution is not sensitive to laser intensities, which is different from the one on the polarization plane, and is also opposite with the expectation of the tunneling ionization model 62.

Finally, we study the HHG process. Fig. 4 shows our results for the radiation process of HHG. Through the comparison between the results without the Coulomb interaction, we found the $\mathrm{HHG}$ process is insensitive to the Coulomb interaction, which is very different from the ATI process. Since the radiation of dipole moment happens near the ion core, the Coulomb interaction in the outer region is not important. As expected, the HHG for the circularly polarized laser is highly suppressed.

\section{CONCLUSION}

We performed a comprehensive study on the interaction between a hydrogen atom and a strong elliptical laser field by solving the TDSE3D, and mainly focused on the above-threshold ionization and high-order harmonic generation. Based on our TDSE3D results, we suggested a further improvement of the modified PPT theory [54] for the total ionization probability by incorporating the barrier-suppression correction used in the modified ADK theory 26]. Similar to the case of the weak field 1, 2, an

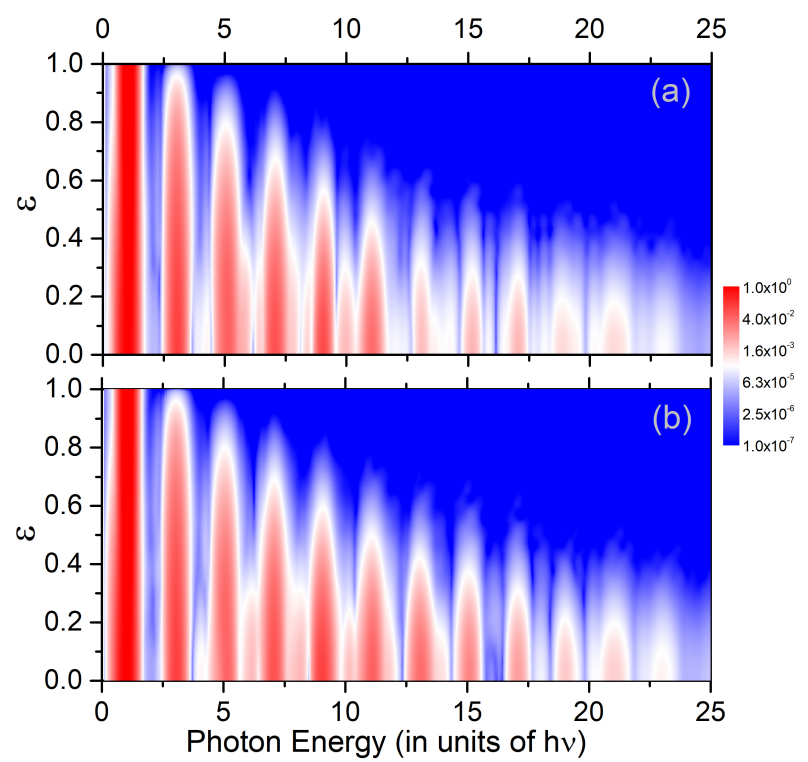

FIG. 4. (Color online) HHG spectra of hydrogen atoms in elliptical laser fields as a function of the ellipticity with (a) and without (b) the Coulomb interaction. The laser parameters are the same as those in Fig. 2 .

intimate relation between bound state excitation probabilities and the ionization probabilities still exists in the strong laser field, and the relation does not depend on the ellipticity. The comparison between TDSE3D calculations with and without the long-range Coulomb interaction could check the validity of the SFA [17- 20]. The long-range Coulomb interaction is found to have crucial influence on the ATI process, but have negligible influence on the HHG process. Furthermore, we investigated the $1 \mathrm{D}$ momentum distribution along the direction perpendicular to the polarization plane and concluded that the interesting variations with the increasing ellipticity of the 1D momentum distribution is due to the Coulomb focusing effect 64]. The distribution is also found to be not sensitive to the laser intensities.

\section{ACKNOWLEDGMENT}

This work was supported by a Grant-in-Aid for Scientific Research (Grant No. JP16K05495) from the Japan Society for the Promotion of Science. The calculations were performed using Oakforest-Pacs at Center for Computational Sciences, University of Tsukuba. 
[1] U. Fano and J. W. Cooper, "Spectral distribution of atomic oscillator strengths," Rev. Mod. Phys. 40, 441507 (1968)

[2] M J Seaton, "Quantum defect theory," Rep. Prog. Phys. 46, 167 (1983)

[3] Mireille Aymar, Chris H. Greene, and Eliane LucKoenig, "Multichannel rydberg spectroscopy of complex atoms," Rev. Mod. Phys. 68, 1015-1123 (1996).

[4] Xiang Gao, Xiao-Ying Han, and Jia-Ming Li, "Intimate relationship between spectroscopy and collisions: a scenario to calculate relevant atomic data for astrophysics," J. Phys. B: At., Mol. Opt. Phys. 49, 214005 (2016).

[5] P. Agostini, F. Fabre, G. Mainfray, G. Petite, and N. K. Rahman, "Free-free transitions following six-photon ionization of xenon atoms," Phys. Rev. Lett. 42, 1127-1130 (1979)

[6] R. R. Freeman, P. H. Bucksbaum, H. Milchberg, S. Darack, D. Schumacher, and M. E. Geusic, "Abovethreshold ionization with subpicosecond laser pulses," Phys. Rev. Lett. 59, 1092-1095 (1987)

[7] A. McPherson, G. Gibson, H. Jara, U. Johann, T. S. Luk, I. A. McIntyre, K. Boyer, and C. K. Rhodes, "Studies of multiphoton production of vacuum-ultraviolet radiation in the rare gases," J. Opt. Soc. Am. B 4, 595-601 (1987)

[8] X. F. Li, A. L'Huillier, M. Ferray, L. A. Lompre, and G. Mainfray, "Multiple-harmonic generation in rare gases at high laser intensity," Phys. Rev. A 39, 5751-5761 (1989)

[9] J. Itatani, D. Zeidler, J. Levesque, M. Spanner, D. M. Villeneuve, and P. B. Corkum, "Controlling high harmonic generation with molecular wave packets," Phys. Rev. Lett. 94, 123902 (2005)

[10] J. Itatani, J. Levesque, D. Zeidler, Hiromichi Niikura, H. Pepin, J. C. Kieffer, P. B. Corkum, and D. M. Villeneuve, "Tomographic imaging of molecular orbitals," Nature 432, 867-871 (2004)

[11] Manfred Lein, "Molecular imaging using recolliding electrons," J. Phys. B: At. Mol. Opt. Phys. 40, R135 (2007)

[12] Moritz Meckel, D Comtois, D Zeidler, Andre Staudte, D Pavičić, HC Bandulet, H Pépin, JC Kieffer, R Dörner, DM Villeneuve, et al., "Laser-induced electron tunneling and diffraction," Science 320, 1478-1482 (2008)

[13] Z. Chang, A. Rundquist, H. Wang, M. M. Murnane, and H. C. Kapteyn, "Generation of coherent soft x-rays at 2.7 nm using high harmonics," Phys. Rev. Lett. 79, 29672970 (1997)

[14] Juan J. Carrera, X. M. Tong, and Shih-I Chu, "Creation and control of a single coherent attosecond xuv pulse by few-cycle intense laser pulses," Phys. Rev. A 74, 023404 (2006)

[15] Ferenc Krausz and Misha Ivanov, "Attosecond physics," Rev. Mod. Phys. 81, 163-234 (2009).

[16] P. B. Corkum, "Plasma perspective on strong field multiphoton ionization," Phys. Rev. Lett. 71, 1994-1997 (1993)

[17] M. Lewenstein, Ph. Balcou, M. Yu. Ivanov, Anne L'Huillier, and P. B. Corkum, "Theory of high-harmonic generation by low-frequency laser fields," Phys. Rev. A 49, 2117-2132 (1994).

[18] Anne L'Huillier, M. Lewenstein, P. Salières, Ph. Balcou, M. Yu. Ivanov, J. Larsson, and C. G. Wahlström,
"High-order harmonic-generation cutoff," Phys. Rev. A 48, R3433-R3436 (1993)

[19] A Becker and F H M Faisal, "Intense-field many-body s-matrix theory," J. Phys. B: At. Mol. Opt. Phys. 38, R1-R56 (2005).

[20] Anh-Thu Le, Hui Wei, Cheng Jin, and C D Lin, "Strongfield approximation and its extension for high-order harmonic generation with mid-infrared lasers," J. Phys. B: At. Mol. Opt. Phys. 49, 053001 (2016)

[21] L. V. Keldysh, "Ionization in the field of a strong electromagnetic wave," Sov. JETP 47, 1945-1950 (1964)

[22] A. M. Perelomov, V. S. Popov, and M. V. Terent'ev, "Ionization of atoms in an alternating electric field," Sov. JETP 23, 924-934 (1966).

[23] M. V. Ammosov, N. B. Delone, and V. P. Krainov, "Tunnel ionizaiton of complex atoms and of atomic ions in an alternating electromagnetic field," Sov. Phys. JETP 64, 1191-1194 (1986)

[24] F H M Faisal, "Multiple absorption of laser photons by atoms," J. Phys. B: 6, L89 (1973).

[25] Howard R. Reiss, "Effect of an intense electromagnetic field on a weakly bound system," Phys. Rev. A 22, 1786$1813(1980)$.

[26] X M Tong and C D Lin, "Empirical formula for static field ionization rates of atoms and molecules by lasers in the barrier-suppression regime," J. Phys. B: At. Mol. Opt. Phys. 38, 2593 (2005)

[27] S. I. Chu and J. Cooper, "Threshold shift and above threshold multiphoton ionization of atomic hydrogen in intense laser fields," Phys. Rev. A 32, 2769 (1985).

[28] P G Burke, P Francken, and C J Joachain, "R-matrixfloquet theory of multiphoton processes," J. Phys. B: At., Mol. Opt. Phys. 24, 761-790 (1991)

[29] Gerd van de Sand and Jan M. Rost, "Semiclassical description of multiphoton processes," Phys. Rev. A 62, $053403(2000)$

[30] C R Feeler and R E Olson, "Single ionization of ne by intense laser fields," J. Phys. B: At., Mol. Opt. Phys. 33, 1997-2003 (2000)

[31] James S. Cohen, "Reexamination of over-the-barrier and tunneling ionization of the hydrogen atom in an intense field," Phys. Rev. A 64, 043412 (2001).

[32] Kenneth C. Kulander, "Multiphoton ionization of hydrogen: A time-dependent theory," Phys. Rev. A 35, 445447 (1987)

[33] K. J. Schafer and K. C. Kulander, "Energy analysis of time-dependent wave functions: Application to above-threshold ionization," Phys. Rev. A 42, 5794-5797 (1990)

[34] J S Parker, B J S Doherty, K J Meharg, and K T Taylor, "Time delay between singly and doubly ionizing wavepackets in laser-driven helium," J. Phys. B: At. Mol. Opt. Phys. 36, L393-L400 (2003).

[35] E Cormier and P Lambropoulos, "Above-threshold ionization spectrum of hydrogen using b-spline functions," J. Phys. B: At., Mol. Opt. Phys. 30, 77-91 (1997)

[36] S. Dionissopoulou, Th. Mercouris, A. Lyras, and C. A. Nicolaides, "Strong laser-field effects in hydrogen: Highorder above-threshold ionization and photoelectron angular distributions," Phys. Rev. A 55, 4397-4406 (1997) 
[37] Xiao-Min Tong and Shih-I Chu, "Theoretical study of multiple high-order harmonic generation by intense ultrashort pulsed laser fields: A new generalized pseudospectral time-dependent method," Chem. Phys. 217, 119 130 (1997)

[38] Shaohao Chen, Xiang Gao, Jiaming Li, Andreas Becker, and Agnieszka Jaron-Becker, "Application of a numerical-basis-state method to strong-field excitation and ionization of hydrogen atoms," Phys. Rev. A 86, $013410(2012)$

[39] Xiao-Min Tong, "A three-dimensional time-dependent schrödinger equation solver: an application to hydrogen atoms in an elliptical laser field," J. Phys. B: At., Mol. Opt. Phys. 50, 144004 (2017)

[40] André D. Bandrauk and Hai Shen, "Exponential split operator methods for solving coupled time-dependent schrödinger equations," J. Chem. Phys. 99, 1185-1193 (1993)

[41] K Sasaki, X M Tong, and N Toshima, "Coulomb focusing effect on the space distribution of the rescattering electron wavepacket in the laser-atom interaction," J. Phys. B: At., Mol. Opt. Phys. 42, 165603 (2009)

[42] Xiao-Min Tong and Shih-I Chu, "Time-dependent approach to high-resolution spectroscopy and quantum dynamics of rydberg atoms in crossed magnetic and electric fields," Phys. Rev. A 61, 031401 (2000)

[43] S. Borbély, X.-M. Tong, S. Nagele, J. Feist, I. Březinová, F. Lackner, L. Nagy, K. Tőkési, and J. Burgdörfer, "Electron correlations in the antiproton energy-loss distribution in he," Phys. Rev. A 98, 012707 (2018).

[44] C. I. Blaga, F. Catoire, P. Colosimo, G. G. Paulus, H. G. Muller, P. Agostini, and L. F. DiMauro, "Strongfield photoionization revisited," Nature phyics 5, 335-338 (2009)

[45] W. Quan, Z. Lin, M. Wu, H. Kang, H. Liu, X. Liu, J. Chen, J. Liu, X. T. He, S. G. Chen, H. Xiong, L. Guo, H. Xu, Y. Fu, Y. Cheng, and Z. Z. Xu, "Classical aspects in above-threshold ionization with a midinfrared strong laser field," Phys. Rev. Lett. 103, 093001 (2009).

[46] Daniel D. Hickstein, Predrag Ranitovic, Stefan Witte, Xiao-Min Tong, Ymkje Huismans, Paul Arpin, Xibin Zhou, K. Ellen Keister, Craig W. Hogle, Bosheng Zhang, Chengyuan Ding, Per Johnsson, N. Toshima, Marc J. J. Vrakking, Margaret M. Murnane, and Henry C. Kapteyn, "Direct visualization of laser-driven electron multiple scattering and tunneling distance in strong-field ionization," Phys. Rev. Lett. 109, 073004 (2012)

[47] Dmitry A. Telnov and Shih-I Chu, "Low-energy structure of above-threshold-ionization electron spectra: Role of the coulomb threshold effect," Phys. Rev. A 83, 063406 (2011).

[48] Christoph Lemell, Konstantinos I. Dimitriou, Xiao-Min Tong, Stefan Nagele, Daniil V. Kartashov, Joachim Burgdörfer, and Stefanie Gräfe, "Low-energy peak structure in strong-field ionization by midinfrared laser pulses: Two-dimensional focusing by the atomic poten- tial," Phys. Rev. A 85, 011403 (2012)

[49] Christoph Lemell, Joachim Burgdörfer, Stefanie Gräfe, Konstantinos I. Dimitriou, Diego G. Arbó, and XiaoMin Tong, "Classical-quantum correspondence in atomic ionization by midinfrared pulses: Multiple peak and interference structures," Phys. Rev. A 87, 013421 (2013)

[50] Xiao-Min Tong, Predrag Ranitovic, Daniel D. Hickstein, Margaret M. Murnane, Henry C. Kapteyn, and Nobuyuki Toshima, "Enhanced multiple-scattering and intra-half-cycle interferences in the photoelectron angular distributions of atoms ionized in midinfrared laser fields," Phys. Rev. A 88, 013410 (2013).

[51] Xiao-Min Tong and Shih-I Chu, "Density-functional theory with optimized effective potential and self-interaction correction for ground states and autoionizing resonances," Phys. Rev. A 55, 3406-3416 (1997).

[52] X. M. Tong, K. Hino, and N. Toshima, "Phasedependent atomic ionization in few-cycle intense laser fields," Phys. Rev. A 74, 031405 (2006).

[53] C. Hofmann, T. Zimmermann, A. Zielinski, and A. S. Landsman, New Journal of Physics 18, 043011 (2016).

[54] S. V. Popruzhenko, V. D. Mur, V. S. Popov, and D. Bauer, "Strong field ionization rate for arbitrary laser frequencies," Phys. Rev. Lett. 101, 193003 (2008).

[55] Xiang Gao, Shao-Hao Chen, and Jia-Ming Li, "Finite space complete basis method: Precision computation of high-resolution spectrum near ionization threshold," Chin. Phys. Lett. 26, 013102 (2009)

[56] A. S. Alnaser, X. M. Tong, T. Osipov, S. Voss, C. M. Maharjan, B. Shan, Z. Chang, and C. L. Cocke, "Laserpeak-intensity calibration using recoil-ion momentum imaging," Phys. Rev. A 70, 023413 (2004)

[57] L. Arissian, C. Smeenk, F. Turner, C. Trallero, A. V. Sokolov, D. M. Villeneuve, A. Staudte, and P. B. Corkum, "Direct test of laser tunneling with electron momentum imaging," Phys. Rev. Lett. 105, 133002 (2010)

[58] C. Smeenk, J. Z. Salvail, L. Arissian, P. B. Corkum, C. T. Hebeisen, and A. Staudte, "Precise in-situ measurement of laser pulse intensity using strong field ionization," Opt. Express 19, 9336-9344 (2011)

[59] I. A. Ivanov, Phys. Rev. A 90, 013418 (2014)

[60] I. A. Ivanov, Phys. Rev. A 93, 053403 (2016)

[61] I. A. Ivanov, A. S. Kheifets, J. E. Calvert, S. Goodall, X. Wang, H. Xu, A. J. Palmer, D. Kielpinski, I. V. Litvinyuk, and R. T. Sang, Scientific Reports 6, 19002 (2016)

[62] N B Delone and Vladimir P Krainov, "Tunneling and barrier-suppression ionization of atoms and ions in a laser radiation field," Physics-Uspekhi 41, 469 (1998)

[63] D Kielpinski, R T Sang, and I V Litvinyuk, "Benchmarking strong-field ionization with atomic hydrogen," Journal of Physics B: Atomic, Molecular and Optical Physics 47, 204003 (2014).

[64] T. Brabec, M. Y. Ivanov, and P. B. Corkum, "Coulomb focusing in intense field atomic processes," Phys. Rev. A 54, R2551-R2554 (1996) 